\title{
A Convenient Adomian-Pade Technique for the Nonlinear Oscillator Equation
}

\author{
Na Wei, ${ }^{1}$ Cheng-Xi Liu, ${ }^{2}$ Teng Hu, ${ }^{2}$ and Fu-Cai You ${ }^{3}$ \\ ${ }^{1}$ State Key Laboratory of Oil and Gas Reservoirs Geology and Exploration, Southwest Petroleum University, Chengdu 610500, China \\ ${ }^{2}$ Key Laboratory of Numerical Simulation of Sichuan Province and College of Mathematics and Information Science, \\ Neijiang Normal University, Neijiang 641112, China \\ ${ }^{3}$ Department of Basic Sciences, Shenyang Institute of Engineering, Shenyang 110136, China
}

Correspondence should be addressed to Cheng-Xi Liu; liuchengxi050317@163.com

Received 26 February 2014; Revised 12 March 2014; Accepted 12 March 2014; Published 9 April 2014

Academic Editor: Dumitru Baleanu

Copyright (C) $2014 \mathrm{Na}$ Wei et al. This is an open access article distributed under the Creative Commons Attribution License, which permits unrestricted use, distribution, and reproduction in any medium, provided the original work is properly cited.

Very recently, the convenient way to calculate the Adomian series was suggested. This paper combines this technique and the Pade approximation to develop some new iteration schemes. Then, the combined method is applied to nonlinear models and the residual functions illustrate the accuracies and conveniences.

\section{Introduction}

Analytical methods for nonlinear systems have caught much attention due to their convenience for obtaining solutions in real engineering problems. One of the most often used methods is the Adomian decomposition method (ADM) [1]. Also due to the rapid development of the computer science, various modifications of these nonlinear analytical methods have been proposed and have been extensively applied to various nonlinear systems [2-14].

Very recently, for the ADM, Duan [4-6] suggested a convenient Adomian calculation scheme. The method can help us get a higher accuracy and can hand higher order approximation problem due to its easier calculation of the Adomian series than the classical one [1]. The technique has been successfully extended to fractional differential equations and boundary value problems.

Recently, Tsai and Chen [11] proposed a Laplace-Adomian-Pade method (LAPM). The method holds the following merits: (a) Laplace transform can be used to determine the initial iteration value; (b) the Pade technique is adopted to accelerate the convergence.
With Duan and Tsai's idea, this paper suggests a novel approximation scheme for the oscillating physical mechanism of the nonlinear models [15]

$$
\begin{aligned}
& \frac{d T}{d t}=C T+D h-\varepsilon T^{3}, \quad T(0)=1, \\
& \frac{d h}{d t}=-E T-R_{h} h, \quad h(0)=1,
\end{aligned}
$$

where $C, D, E$, and $R_{h}$ are physical constants, $T$ describes the temperature of the eastern equatorial Pacific sea surface, and $h$ is the thermocline depth anomaly.

\section{Preliminaries of the Adomian Series}

Generally, consider the following nonlinear equation:

$$
L[u]+R[u]+N[u]=g(t),
$$

where $L$ is the highest derivative of $u$, for example, $m$ order, $R$ is the remaining linear part containing the lower order derivatives, and $N$ is the nonlinear operator. 
Apply the inverse $L^{-1}$ of the linear operator $L$ in (2), and we can obtain

$$
\begin{aligned}
u= & u(0)+u^{\prime}(0) t+\cdots+u^{(m-1)}(0) \frac{t^{m-1}}{(m-1) !} \\
& +L^{-1}(g(t))-L^{-1}(R[u])-L^{-1}(N[u]) .
\end{aligned}
$$

Consider the basic idea of the Picard method

$$
\begin{aligned}
u_{n+1}= & u(0)+u^{\prime}(0) t+\cdots+u^{(m-1)}(0) \frac{t^{m-1}}{(m-1) !} \\
& +L^{-1}(g(t))-L^{-1}\left(R\left[u_{n}\right]\right)-L^{-1}\left(N\left[u_{n}\right]\right),
\end{aligned}
$$

and assume that

$$
u=\sum_{i=0}^{\infty} v_{i}, \quad u_{n}=\sum_{i=0}^{n} v_{i} .
$$

The classical ADM [1] supposes that the nonlinear term $N[u]$ can be expanded approximately as

$$
N[u]=\sum_{n=0}^{\infty} A_{n}
$$

where $A_{n}$ is calculated by

$$
A_{n}=\frac{1}{n !} \frac{\partial^{n}}{\partial \lambda^{n}}\left[N\left(\sum_{k=0}^{\infty} v_{k} \lambda^{k}\right)\right]_{\lambda=0} .
$$

For example, $\sum_{n=0}^{\infty} A_{n}$ is the Adomian series of $T^{3}$; namely,

$$
\begin{aligned}
& A_{0}=v_{0}^{3}, \\
& A_{1}=3 v_{0}^{2} v_{1}, \\
& A_{2}=3 v_{0} v_{1}^{2}+3 v_{0}^{2} v_{2},
\end{aligned}
$$

Duan et al. [4-6] very recently suggested a convenient way to calculate the Adomian series as

$$
A_{n}=\frac{1}{n} \sum_{k=0}^{n-1}(k+1) v_{k+1} \frac{d A_{n-1-k}}{d v_{0}}
$$

as well as the case of the $m$-variable

$$
A_{n}=\frac{1}{n} \sum_{i=1}^{m} \sum_{k=0}^{n-1}(k+1) v_{i, k+1} \frac{\partial A_{n-1-k}}{\partial v_{i, 0}}
$$

For the single variable case, $N[u]=f(u)$, the first three components are listed as

$$
\begin{aligned}
A_{1}= & v_{1} \frac{d f\left(v_{0}\right)}{d v_{0}}, \\
A_{2}= & \frac{1}{2} v_{1}^{2} \frac{d^{2} f\left(v_{0}\right)}{d v_{0}^{2}}+v_{2} \frac{d f\left(v_{0}\right)}{d v_{0}}, \\
A_{3}= & \frac{1}{6} v_{1}{ }^{3} \frac{d^{3} f\left(v_{0}\right)}{d v_{0}^{3}}+v_{2} v_{1} \frac{d^{2} f\left(v_{0}\right)}{d v_{0}^{2}}+v_{3} \frac{d f\left(v_{0}\right)}{d v_{0}}, \\
A_{4}= & \frac{1}{24} v_{1} \frac{d^{4} f\left(v_{0}\right)}{d v_{0}^{4}}+\frac{1}{2} v_{2} v_{1}{ }^{2} \frac{d^{3} f\left(v_{0}\right)}{d v_{0}^{3}} \\
& +v_{3} v_{1} \frac{d^{2} f\left(v_{0}\right)}{d v_{0}^{2}}+\frac{1}{2} v_{2}{ }^{2} \frac{d^{2} f\left(v_{0}\right)}{d v_{0}^{2}} \\
& +v_{4} \frac{d f\left(v_{0}\right)}{d v_{0}} .
\end{aligned}
$$

And for the two-variable case, $N[u]=f\left(u_{1}, u_{2}\right)$, the first three components are listed as

$$
\begin{aligned}
& A_{1}=v_{1,1} \frac{\partial f\left(v_{1,0}, v_{2,0}\right)}{\partial v_{1,0}}+v_{2,1} \frac{\partial f\left(v_{1,0}, v_{2,0}\right)}{\partial v_{2,0}} \\
& A_{2}=\frac{1}{2} v_{1,1}{ }^{2} \frac{\partial^{2} f\left(v_{1,0}, v_{2,0}\right)}{\partial v_{1,0}{ }^{2}}+v_{1,1} v_{2,1} \frac{\partial^{2} f\left(v_{1,0}, v_{2,0}\right)}{\partial v_{2,0} \partial v_{1,0}} \\
& +v_{1,2} \frac{\partial f\left(v_{1,0}, v_{2,0}\right)}{\partial v_{1,0}}+\frac{1}{2} v_{2,1}{ }^{2} \frac{\partial^{2} f\left(v_{1,0}, v_{2,0}\right)}{\partial v_{2,0}^{2}} \\
& +v_{2,2} \frac{\partial f\left(v_{1,0}, v_{2,0}\right)}{\partial v_{2,0}} \\
& A_{3}=\frac{1}{6} v_{1,1}{ }^{3} \frac{\partial^{3} f\left(v_{1,0}, v_{2,0}\right)}{\partial v_{1,0}{ }^{3}}+\frac{1}{2} v_{1,1}{ }^{2} v_{2,1} \frac{\partial^{3} f\left(v_{1,0}, v_{2,0}\right)}{\partial v_{2,0} \partial v_{1,0}{ }^{2}} \\
& +v_{1,1} v_{1,2} \frac{\partial^{2} f\left(v_{1,0}, v_{2,0}\right)}{\partial v_{1,0}^{2}}+\frac{1}{2} v_{1,1} v_{2,1}{ }^{2} \frac{\partial^{3} f\left(v_{1,0}, v_{2,0}\right)}{\partial v_{2,0}{ }^{2} \partial v_{1,0}} \\
& +v_{1,1} v_{2,2} \frac{\partial^{2} f\left(v_{1,0}, v_{2,0}\right)}{\partial v_{2,0} \partial v_{1,0}}+v_{1,2} v_{2,1} \frac{\partial^{2} f\left(v_{1,0}, v_{2,0}\right)}{\partial v_{2,0} \partial v_{1,0}} \\
& +v_{1,3} \frac{\partial f\left(v_{1,0}, v_{2,0}\right)}{\partial v_{1,0}}+\frac{1}{6} v_{2,1}{ }^{3} \frac{\partial^{3} f\left(v_{1,0}, v_{2,0}\right)}{\partial v_{2,0}{ }^{3}} \\
& +v_{2,1} v_{2,2} \frac{\partial^{2} f\left(v_{1,0}, v_{2,0}\right)}{\partial v_{2,0}^{2}}+v_{2,3} \frac{\partial f\left(v_{1,0}, v_{2,0}\right)}{\partial v_{2,0}} \text {. }
\end{aligned}
$$

The above formulae (9) spend less time deriving the $A_{n}$. On the other hand, this provides a possible tool to investigate the higher order approximation solution. 


\section{Iteration Schemes Based on the Convenient Adomian Series}

Now, we present our analytical schemes using the convenient Adomian series, Laplace transform, and Pade approximation. We adopt the steps in [16]. Considering (2), we show the following iteration schemes.

(i) Take Laplace transform $\widetilde{L}$ to both sides:

$$
\widetilde{L}[L[u]+R[u]]+\widetilde{L}[N[u]]=\widetilde{L}[g(t)] .
$$

We can have iteration formula (4) through inverse of Laplace transform $\widetilde{L}^{-1}$ :

$$
u(t)=f(t)+\widetilde{L}^{-1}[\widetilde{\lambda}(s) \widetilde{L}[N[u]]],
$$

where $f(t)$ and $\widetilde{\lambda}(s)$ can be determined by calculation of Laplace transform to $L[u], R[u]$, and $g(t)$. The calculation of $\tilde{\lambda}(s)$ is similar to the determination of the Lagrange multiplier of the variational iteration method in [17].

(ii) Through the Picard successive approximation, we can obtain the following iteration formula:

$$
u_{n+1}=f(t)+\widetilde{L}^{-1}\left[\tilde{\lambda}(s) \widetilde{L}\left[N\left[u_{n}\right]\right]\right] .
$$

(iii) Let $u_{n}=\sum_{i=0}^{n} v_{i}$ and apply the Adomian series to expand the term $N[u]$ as $\sum_{i=0}^{\infty} A_{i}$. Then, the iteration formula reads

$$
\begin{gathered}
v_{n+1}=\widetilde{L}^{-1}\left[\tilde{\lambda}(s) \widetilde{L}\left[A_{n}\right]\right], \\
v_{0}=f(t),
\end{gathered}
$$

where $A_{i}$ are calculated by

$$
A_{n}=\frac{1}{n} \sum_{k=0}^{n-1}(k+1) v_{k+1} \frac{d A_{n-1-k}}{d v_{0}} .
$$

(iv) Employ the Pade technique to accelerate the convergence of $u_{n}=\sum_{i=0}^{n} v_{i}$.

\section{Applications of the Iteration Formulae}

In this study, we consider a reduced case where $D=0$ and $0<\varepsilon \ll 1$ in (1) as follows:

$$
\frac{d T}{d t}=C T-\varepsilon T^{3}, \quad C=1, T(0)=1 .
$$

In order to solve (18) with the Maple software, apply Laplace transform $L$ to both sides firstly. This step can fully and optimally determine the initial iteration. We can derive

$$
\begin{gathered}
v_{n+1}(t)=-\widetilde{L}^{-1}\left[\frac{\varepsilon \widetilde{L}\left[A_{n}\right]}{(s-C)}\right], \quad n \geq 1, \\
v_{0}=\widetilde{L}^{-1}\left[\frac{T(0)}{(s-C)}\right] .
\end{gathered}
$$

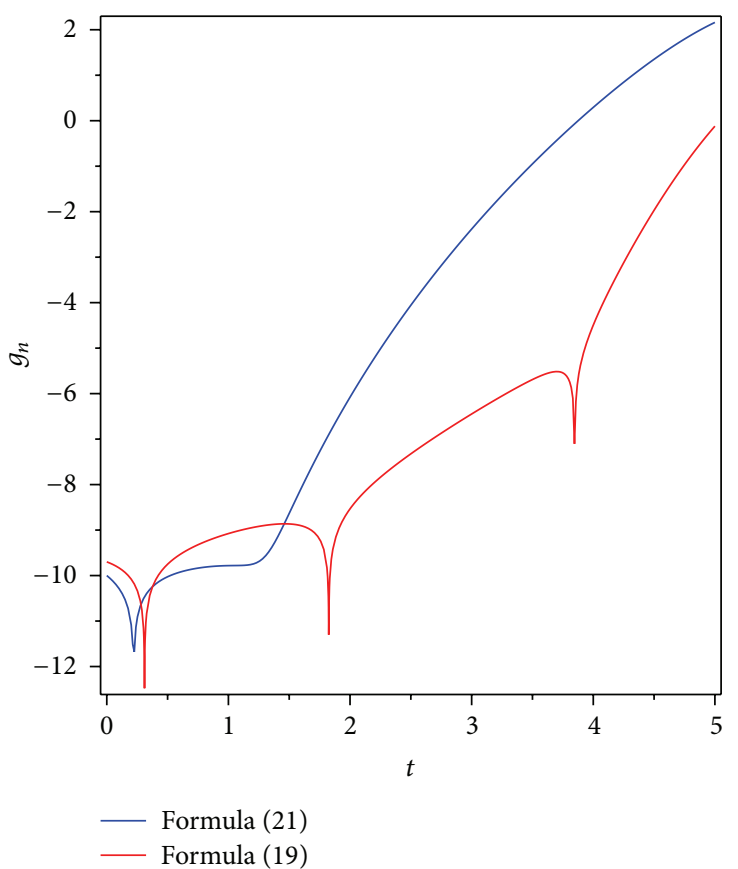

FIGURE 1: The comparisons of the approximate solutions using (19) and (21).

Setting $\varepsilon=0.00001$ in the model (18), now we can obtain the first few as

$$
\begin{gathered}
T_{0}=v_{0}=\mathrm{e}^{t}, \\
T_{1}=v_{0}+v_{1}=\mathrm{e}^{t}-0.0001 \mathrm{e}^{2 t} \sinh (t),
\end{gathered}
$$

Apply the Pade technique to $T_{n}$ and denote the result as $P T_{1, n}[p / q]$.

We now can compare the accuracies of the different versions of the Adomian decomposition methods.

For example, we can write out the classical Adomian formula for (18) as

$$
\begin{gathered}
v_{n+1}(t)=C \int_{0}^{t} v_{n} d \tau-\varepsilon \int_{0}^{t} A_{n} d \tau, \quad n \geq 1, \\
v_{0}=T(0) .
\end{gathered}
$$

Also apply the Pade technique to $T_{n}$ and denote the result as $P T_{2, n}[p / q]$.

Define the residual function as

$$
\begin{gathered}
g_{n}=\log \mid \frac{d\left(P T_{i, n}[p / q]\right)}{d t}-C\left(P T_{i, n}\left[\frac{p}{q}\right]\right) \\
+\varepsilon\left(P T_{i, n}\left[\frac{p}{q}\right]\right)^{3} \mid, \quad i=1,2 .
\end{gathered}
$$

Consider the same $n=20$ and $p=q=20$; from the comparison illustrated through Figure 1, we can see that 


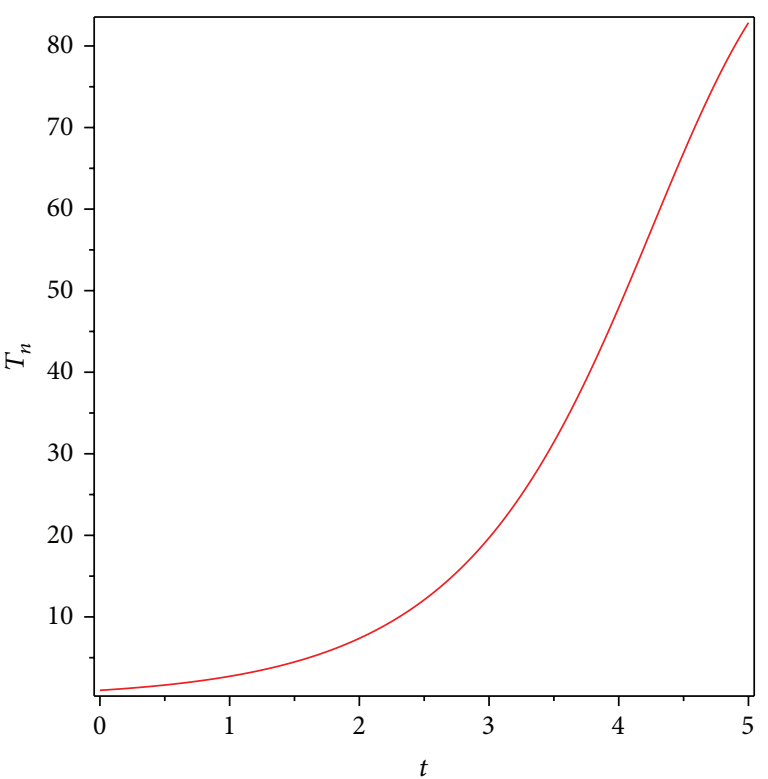

Figure 2: Analytical solution of (18) via (19).

the iteration formula (19) has a higher accuracy almost in the interval $[0,5]$.

As a result, we decide to adopt the iteration formula (19) and give the numerical simulation of (18) in the case of the higher order approximation. The analytical solution is illustrated in Figure 2.

The approximate solution is reliable from the error analysis of the iteration formula (19) in Figure 1.

\section{Conclusions}

The approximate solution is compared with the nonlinear techniques in higher order iteration and the result shows the new way's higher accuracy to calculate the Adomian series. In view of this point, the comparison of different versions of the Adomian method is possible. The results show that the iteration formula fully using all the linear parts has a higher accuracy. It provides an efficient tool to select a suitable algorithm when solving engineering problems.

\section{Conflict of Interests}

The authors declare that there is no conflict of interests regarding the publication of this paper.

\section{Acknowledgments}

This work is supported by the Open Fund of State Key Laboratory of Oil and Gas Geology and Exploration, Southwest Petroleum University (PLN1309), National Natural Science Foundation of China "Study on wellbore flow model in liquid-based whole process underbalanced drilling" (Grant No. 51204140), the Scientific Research Fund of Sichuan
Provincial Education Department (4ZA0244), and the Program for Liaoning Excellent Talents in University under Grant no. LJQ2011136.

\section{References}

[1] G. Adomian, Solving Frontier Problems of Physics: the Decomposition Method, Kluwer Academic Publishers, Boston, Mass, USA, 1994.

[2] S. S. Ray and R. K. Bera, "An approximate solution of a nonlinear fractional differential equation by Adomian decomposition method," Applied Mathematics and Computation, vol. 167, no. 1, pp. 561-571, 2005.

[3] H. Jafari and V. Daftardar-Gejji, "Revised Adomian decomposition method for solving a system of nonlinear equations," Applied Mathematics and Computation, vol. 175, no. 1, pp. 1-7, 2006.

[4] J.-S. Duan, "Recurrence trianglefor Adomian polynomials," Applied Mathematics and Computation, vol. 216, no. 4, pp. 12351241, 2010.

[5] J.-S. Duan, "An efficient algorithm for the multivariable Adomian polynomials," Applied Mathematics and Computation, vol. 217 , no. 6, pp. 2456-2467, 2010.

[6] J. S. Duan, R. Rach, D. Baleanu, and A. M. Wazwaz, "A review of the Adomian decomposition method and its applications to fractional differential equations," Communications in Fractional Calculus, vol. 3, no. 2, pp. 73-99, 2012.

[7] G.-C. Wu, "Adomian decomposition method for non-smooth initial value problems," Mathematical and Computer Modelling, vol. 54, no. 9-10, pp. 2104-2108, 2011.

[8] V. Daftardar-Gejji and H. Jafari, "Adomian decomposition: a tool for solving a system of fractional differential equations," Journal of Mathematical Analysis and Applications, vol. 301, no. 2, pp. 508-518, 2005.

[9] H. Jafari and V. Daftardar-Gejji, "Solving linear and nonlinear fractional diffusion and wave equations by Adomian decomposition," Applied Mathematics and Computation, vol. 180, no. 2, pp. 488-497, 2006.

[10] S. Momani and Z. Odibat, "Analytical solution of a timefractional Navier-Stokes equation by Adomian decomposition method," Applied Mathematics and Computation, vol. 177, no. 2, pp. 488-494, 2006.

[11] P.-Y. Tsai and C.-K. Chen, "An approximate analytic solution of the nonlinear Riccati differential equation," Journal of the Franklin Institute, vol. 347, no. 10, pp. 1850-1862, 2010.

[12] D. Q. Zeng and Y. M. Qin, "The Laplace-Adomian-Pade technique for the seepage flows with the Riemann-Liouville derivatives," Communications in Fractional Calculus, vol. 3, no. 1, pp. 26-29, 2012.

[13] H. Jafari, C. M. Khalique, and M. Nazari, "Application of the Laplace decomposition method for solving linear and nonlinear fractional diffusionwave equations," Applied Mathematics Letters, vol. 24, no. 11, pp. 1799-1805, 2011.

[14] H. Jafari, M. Nazari, D. Baleanu, and C. M. Khalique, "A new approach for solving a system of fractional partial differential equations," Computers \& Mathematics with Applications, vol. 66, no. 5, pp. 838-843, 2013.

[15] J. Q. Mo and W.-T. Lin, "Generalized variation iteration solution of an atmosphere-ocean oscillator model for global climate," Journal of Systems Science and Complexity, vol. 24, no. 2, pp. 271276, 2011. 
[16] Y. Zeng, "The Laplace-Adomian-Pade technique for the ENSO model," Mathematical Problems in Engineering, vol. 2013, Article ID 954857, 4 pages, 2013.

[17] G. C. Wu, "Challenge in the variational iteration methoda new approach to identification of the Lagrange multipliers," Journal of King Saud University -Science, vol. 25, no. 2, pp. 175178, 2013. 


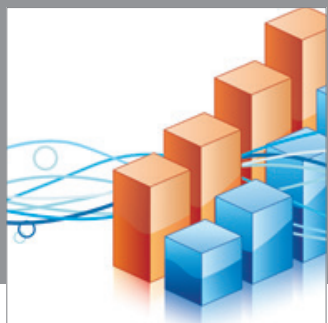

Advances in

Operations Research

mansans

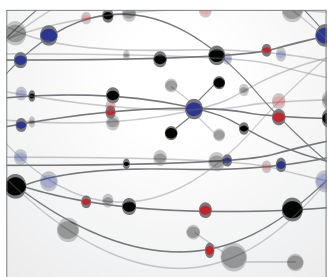

The Scientific World Journal
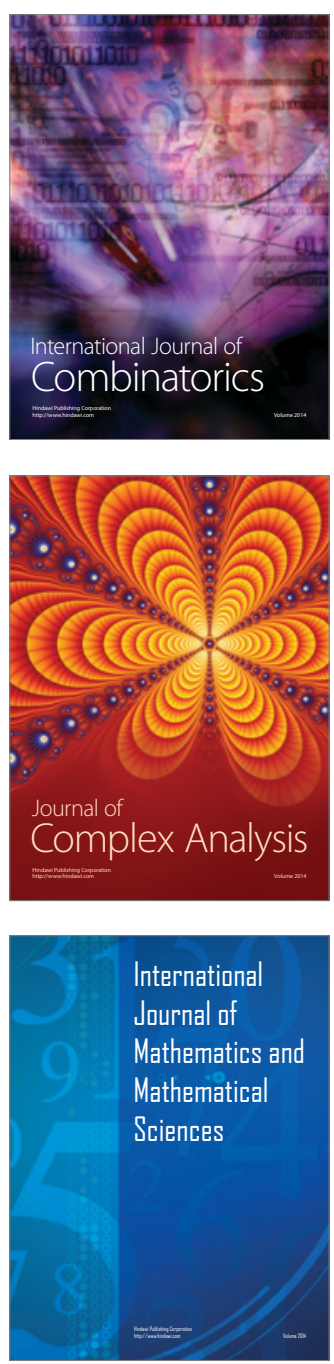
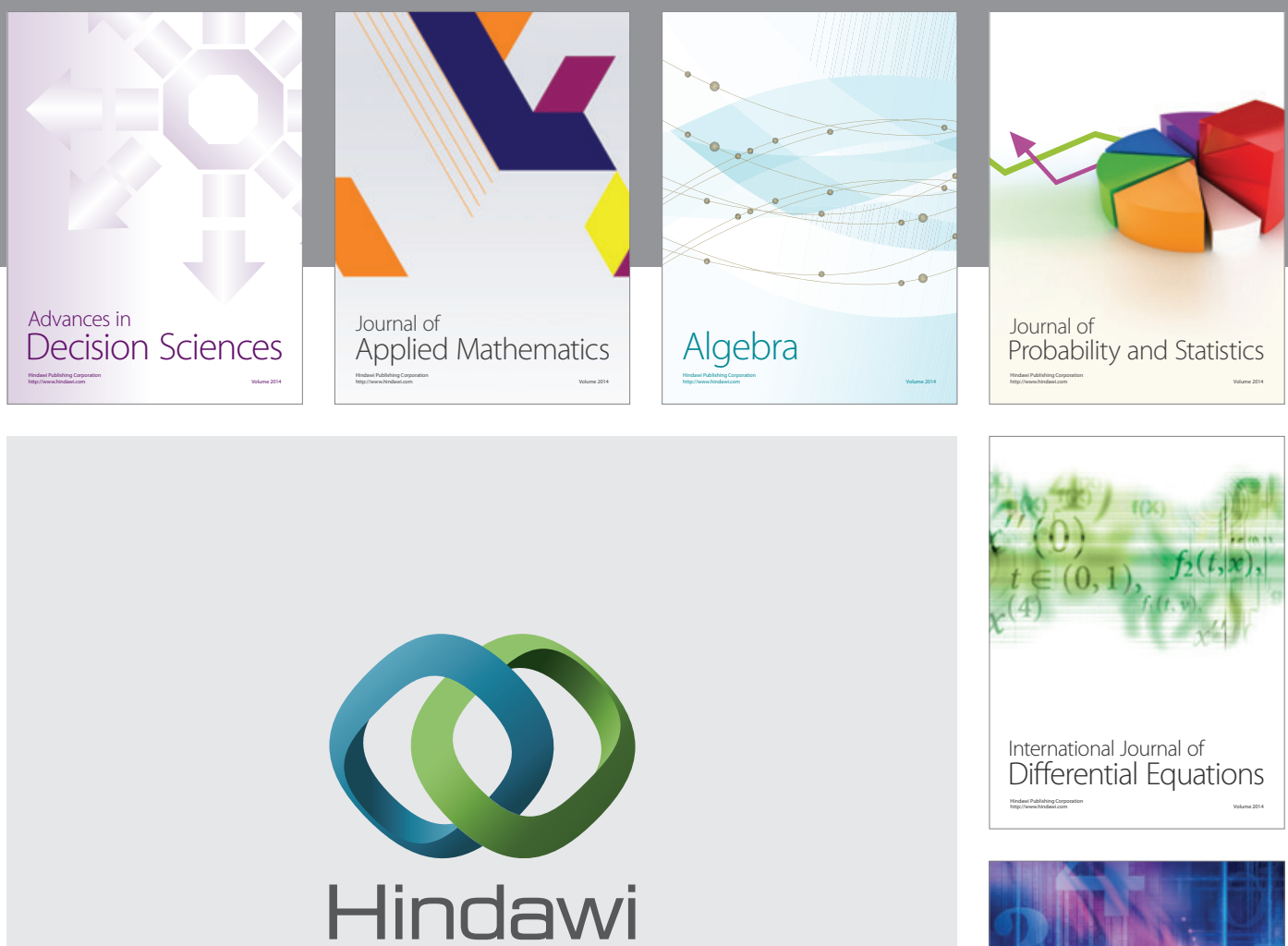

Submit your manuscripts at http://www.hindawi.com
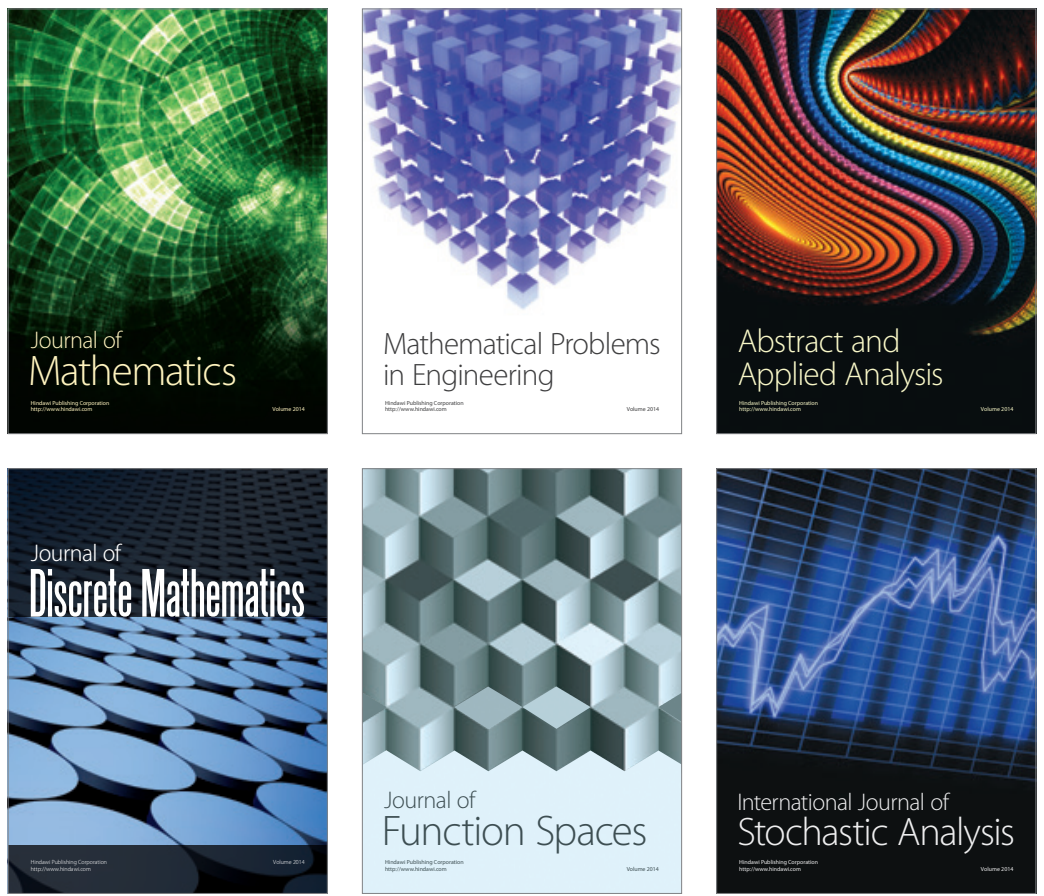

Journal of

Function Spaces

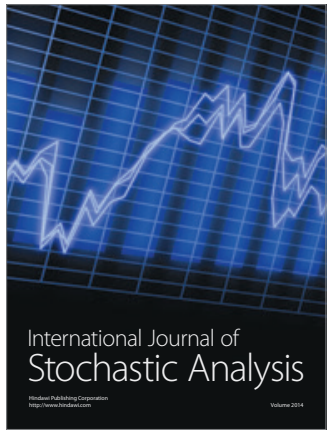

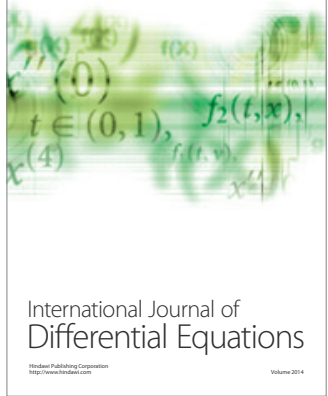
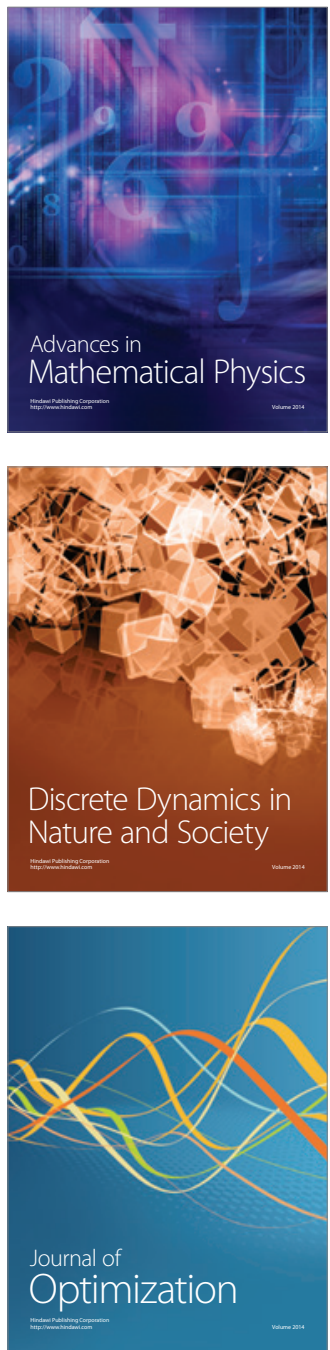